TITLE:

\title{
Single-shot ultrafast electron diffraction with a laser-accelerated sub-MeV electron pulse
}

\section{$\operatorname{AUTHOR}(\mathrm{S})$ :}

Tokita, Shigeki; Inoue, Shunsuke; Masuno, Shinichiro; Hashida, Masaki; Sakabe, Shuji

\section{CITATION:}

Tokita, Shigeki ...[et al]. Single-shot ultrafast electron diffraction with a laser-accelerated sub-MeV electron pulse. Applied Physics Letters 2009, 95(11): 111911.

ISSUE DATE:

2009-09

URL:

http://hdl.handle.net/2433/86205

RIGHT:

c 2009 American Institute of Physics. 


\title{
Single-shot ultrafast electron diffraction with a laser-accelerated sub-MeV electron pulse
}

\author{
Shigeki Tokita, ${ }^{a)}$ Shunsuke Inoue, Shinichiro Masuno, Masaki Hashida, and Shuji Sakabe \\ Advanced Research Center for Beam Science, Institute for Chemical Research, Kyoto University, \\ Gokasho, Uji, Kyoto 611-0011, Japan and Department of Physics, Graduate School of Science, \\ Kyoto University, Kitashirakawa, Sakyo, Kyoto 606-7501, Japan
}

(Received 4 June 2009; accepted 24 August 2009; published online 17 September 2009)

\begin{abstract}
We have demonstrated single-shot measurement of electron diffraction patterns for a single-crystal gold foil using $340 \mathrm{keV}$ electron pulses accelerated by intense femtosecond laser pulses with an intensity of $2 \times 10^{18} \mathrm{~W} / \mathrm{cm}^{2}$. The measured electron beam profile is faithfully reproduced by the numerical simulation of the electron trajectory, providing evidence that the electron pulse spontaneously expands in time owing to the velocity spread produced in the acceleration process, but is not distorted in an irreversible nonlinear manner. This study shows that the laser acceleration is promising for the development of pulse compression methods for single-shot femtosecond electron diffraction. (C) 2009 American Institute of Physics. [doi:10.1063/1.3226674]
\end{abstract}

Ultrafast electron diffraction (UED) is a very sensitive and useful method for investigating the transient structures and dynamics of atomic and molecular systems on femtosecond to picosecond time scales. ${ }^{1-4}$ In UED instruments, electron pulses are generated by photoemission with a femtosecond laser and accelerated by a static electric field up to several tens of $\mathrm{keV}$, and consequently a temporal resolution of up to the subpicosecond time scale is demonstrated. However, the highest resolution is obtained only at a low electron flux. This is attributed to electron pulse expansion due to the space-charge effect in the pulse; therefore, the number of electrons per pulse for a given time resolution is limited. ${ }^{5,6}$ Nevertheless, in several recent developments, both the number of electrons and the temporal resolution have been considerably improved to a level that allows the observation of irreversible phenomena with a subpicosecond time resolution. ${ }^{7-9}$ As an example of the most advanced UED technology, Harb et al. ${ }^{8}$ observed an irreversible order-todisorder phase transition of Si crystals using 200 fs electron pulses. The diffraction images presented were averaged over about ten shots, each taken at a fresh sample position. However the space-charge effect is still the most important issue, limiting the number of electrons to less than $10^{4}$ per pulse. As long as a photoemission-based electron source is used without introducing additional techniques, it is no longer likely that the number of electrons can be increased by one order of magnitude to perform single-shot measurements.

Pulse compression for UED is the most promising scheme for overcoming the limitation mentioned above., . $^{20,11}$ By imposing a negative tilt over the length of an electron pulse in its longitudinal phase-space distribution (slow electrons leading to fast electrons), the pulse can be compressed during its flight in free space to achieve a very high electron density at a certain point. Electron pulses with such a phasespace distribution have been demonstrated in relativistic electron accelerators, where either static magnetic fields ${ }^{12}$ or time-dependent electric fields ${ }^{13}$ are employed, and have been applied in $5 \mathrm{MeV}$ UED. ${ }^{14}$ However, energies in the $\mathrm{MeV}$ range are not suitable for general applications due to several

${ }^{a)}$ Electronic mail: tokita@laser.kuicr.kyoto-u.ac.jp. disadvantages such as a rather short de Broglie wavelength, small cross section of elastic scattering, and high radiation damage to samples. A recently proposed scheme to compress self-expanded electron pulses by using the space-charge effect is promising to perform pulse compression in the 100 $\mathrm{keV}$ energy range. ${ }^{11}$ Numerical simulation shows that the self-expanded pulses have a linear positive tilt in the longitudinal phase space, and can be reversed and compressed to less than $100 \mathrm{fs}$ using a radio-frequency (rf) cavity. However, this method has not yet been realized and to determine the actual compressibility, more experimental studies are necessary.

The laser ponderomotive acceleration of electrons in plasma produced by an intense femtosecond laser pulse ${ }^{15-17}$ may be a promising method for generating compressible electron pulses in the sub-MeV range. At intensities approaching $10^{18} \mathrm{~W} / \mathrm{cm}^{2}$, the ponderomotive potential of near-infrared light becomes as high as the relativistic electron energy, and direct acceleration of electrons occurs through interactions with laser fields at the plasma surface. ${ }^{18-20}$ Such an acceleration scheme provides an extremely strong acceleration field compared with conventional electrostatic or RF accelerations. The enormous electric field allows for a marked reduction in the time required for acceleration to levels as short as the laser pulse duration (less than $100 \mathrm{fs}) .^{21}$ More conveniently, laser-accelerated electrons are broadly distributed in energy, and all electrons are accelerated at the same time (in the degree of laser pulse duration). Such an electron pulse rapidly expands in the longitudinal direction during its flight, but the expanding process can be reversed using existing techniques ${ }^{11-13}$ since electrons in a pulse are distributed with a linear tilt in phase space. The following considerations lead us to expect that laser accelerated electron sources have advantages of both compressibility and brightness when compared with conventional photoemission-based electron sources. First, no additional process of energy spreading such as the space-charge effect is required. Thus, the irreversible changes of phase-space structure caused by the nonlinearity of the energy spreading process can be prevented. When the space-charge effect is small enough to be negligible, the phase-space time- 


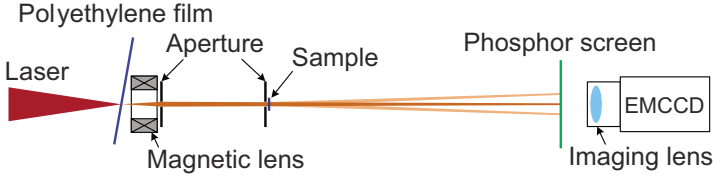

FIG. 1. (Color online) Layout of the electron diffraction experiment.

evolution hardly depends on the conditions of electron pulse generation such as the temporal shape of the laser pulses used to generate electrons, the number of generated electrons, and the transverse spatial distribution of electron emission. This feature will facilitate precise and stable pulse compression. Second, the electron emission flux is not limited by optical damage of the cathode material. Thus, laser-plasma based electron sources have the potential for a radical increase of electron emission flux. For example, increases of laser intensity and pulse energy, ${ }^{16,22}$ control of prelaser pulses, ${ }^{23}$ and optimization of target material and geometry $^{16,24}$ contribute the increase of electron emission flux.

Before focusing on the stage mentioned above, it is important to show that plasma produced with intense laser pulses yields a sufficient number of electrons for single-shot UED. In this paper, sub-MeV electrons are generated from plasma produced by irradiating a tightly focused terawatt femtosecond laser pulse on a solid target. By simply using a magnetic lens, a monoenergetic electron pulse beam with a central energy of about $340 \mathrm{keV}$, which has sufficient beam intensity to take a single-shot diffraction pattern, has been produced.

Electron pulses are generated in the setup shown in Fig. 1. Laser pulses of a Ti:sapphire chirped-pulse amplification system [central wavelength $800 \mathrm{~nm}$, repetition rate $10 \mathrm{~Hz}$, pulse energy $60 \mathrm{~mJ}$, pulse duration $130 \mathrm{fs}$, energy stability $2 \% \mathrm{rms}$ (Ref. 25)] were focused with an $f / 3.5$ off-axis parabolic mirror onto a polyethylene film with a thickness of $12 \mu \mathrm{m}$ in vacuum of $10^{-2} \mathrm{~Pa}$. To prevent the reflected laser pulses from returning into the laser system, the angle of incidence was set at $10^{\circ}$ from the normal. The full width at half maximum (FWHM) and the Strehl ratio of the focused beam were measured to be $4 \mu \mathrm{m}$ and 0.4 , respectively, resulting in an intensity of $2 \times 10^{18} \mathrm{~W} / \mathrm{cm}^{2}$ on the target. Amplified spontaneous emission was estimated to be less than $10^{-7}$ of the peak intensity of the laser pulses. The polyethylene film target with a diameter of $90 \mathrm{~mm}$ is rotated across the laser focus at a velocity of about $5 \mathrm{~mm} / \mathrm{s}$, making it possible to accumulate up to $10^{4}$ shots in a single run. The displacement of the position of the target surface during the operation was actively corrected by a computer-controlled positioning stage to be less than $\pm 5 \mu \mathrm{m}$. A portion of the electrons emitted from the target were collimated with a magnetic lens as- sembled from two circularly symmetric iron pole pieces and a permanent magnet. The magnetic lens had a focal length of about $10 \mathrm{~mm}$ at an electron energy of $340 \mathrm{keV}$. To obtain a low-divergence beam, two apertures with a diameter of $1 \mathrm{~mm}$ were placed behind the magnetic lens at distances of 1.5 and $20 \mathrm{~cm}$ from the target. The sample for electron diffraction was placed immediately behind the second aperture. In order to detect electrons, a phosphor screen and a lenscoupled electron-multiplying charge-coupled device camera (Hamamatsu Photonics, C9100-13) were used. The separation between the sample and the screen was $540 \mathrm{~mm}$. The phosphor screen was made by coating $\mathrm{P} 43$ phosphor powder on a titanium foil of $3 \mu \mathrm{m}$ thickness. The sensitivity of this detection system was calibrated using a commercially available imaging plate (Fujifilm, FDL-UR-V).

Figures 2(a) and 2(c) shows a measured electron beam profile obtained with single-shot laser irradiation in the absence of a sample. The spatial width of the electron beam image was measured to be $0.5 \mathrm{~mm}$ (FWHM). Owing to the very broad momentum distribution of the laser-generated electrons, a "skirt" around the focused beam was formed, and this part included electrons whose momentum was lower or higher than that of the focused beam. The total number of electrons which arrived at the phosphor screen, including the skirt part, was estimated to be $4.2 \times 10^{5}(\sim 0.07 \mathrm{pC})$ from Fig. 2(a). The number of electrons in the focused part, within the FWHM range, was $6 \times 10^{4}(\sim 0.01 \mathrm{pC})$. The shot-to-shot fluctuation in the number of electrons was about $10 \% \mathrm{rms}$ with a laser pulse energy fluctuation of $2 \% \mathrm{rms}$. The number of electrons linearly increased with increasing laser pulse energy from 30 to $60 \mathrm{~mJ}$ without significant increase in the electron beam size. This result suggests that increasing laser energy is one possible way to increase the electron beam intensity. At pulse energies lower than about $30 \mathrm{~mJ}$, the electron beam was not detected in this experiment.

Numerical simulations of the electron trajectories were carried out using the GENERAL PARTICLE TRACER $\operatorname{code}^{26}$ in order to model the electron beam. The space charge effect was not taken into account in the present simulations. The initial momentum and angular distributions of the electrons emitted from the laser-irradiated target were assumed to be uniform. The initial spatial distribution of the emitted electrons is assumed to be Gaussian. When the electrons are initially distributed at a FWHM of $4 \mu \mathrm{m}$, which is as large as the laser focal spot, the calculated electron beam profile at the phosphor screen shows good agreement with the experimental results, as shown in Figs. 2(b) and 2(c). On the other hand, when the electrons are initially distributed at a point, the beam profile shows a much smaller FWHM (about ten times). Therefore the observed beam profile was not determined by aberrations of the magnetic lens. This result proves
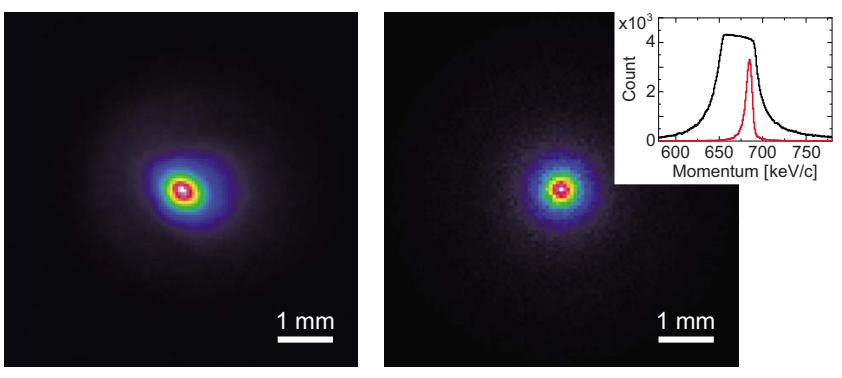

(a)

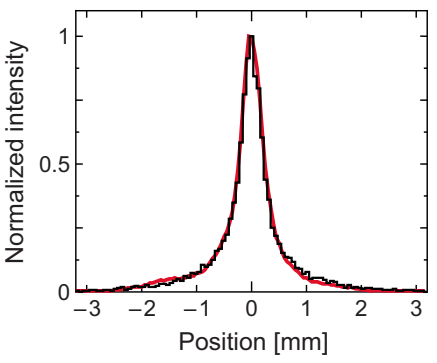

(c)
FIG. 2. (Color online) (a) Measured and (b) calculated electron beam profiles with no sample, obtained by single-shot laser irradiation. (c) Vertical slice through the center of the beam spots on the measured (red line) and calculated (black line) profiles. Inset: Calculated electron momentum distribution of all electrons in the beam profile (black line) and of electrons within the FWHM range of the focused spot (red line). 


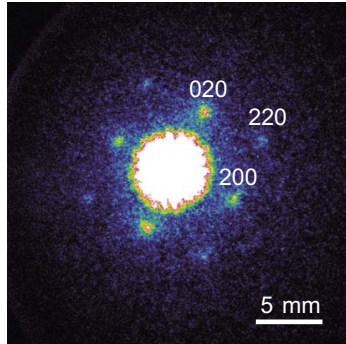

(a)

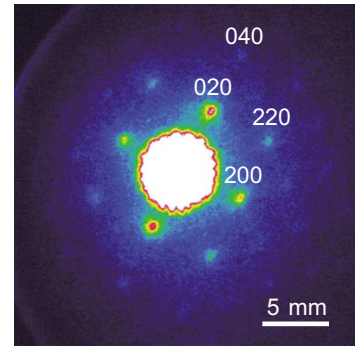

(b)
FIG. 3. (Color online) Measured diffraction pattern obtained from an $\mathrm{Au}$ (001) single-crystal sample with (a) single-shot and (b) 10-shot irradiations.

that the electron emission area is as large as the laserirradiated area. As shown in the inset of Fig. 2(b), we also estimated the momentum distribution based on the simulation result. The momentum spread of all electrons that reached the phosphor screen is as broad as about $10 \%$ of the average momentum. However, since electrons with too high or too low a momentum are not focused on a tight spot at the phosphor screen, a relatively narrow momentum spread can be obtained within the focused spot. The peak momentum and momentum spread of the electrons in the focused part, within the FWHM range, are estimated to be $685 \mathrm{keV} / \mathrm{c}$ (344 $\mathrm{keV}$ in energy) and $1.3 \%$ (FWHM), respectively. From the momentum difference within the focused electron beam, we can estimate the electron pulse duration to be of the order of $10 \mathrm{ps}$ at the sample point.

To demonstrate the imaging of single-shot electron diffraction, diffraction patterns were taken using a 10-nm-thick single-crystal Au foil as a sample. The sample was set on a copper mesh used for general transmission electron microscope observations and was oriented along the (001) zone axis. Figure 3(a) shows a measured single-shot diffraction pattern of the Au sample. Clear diffraction spots of the (020), (200), and (220) planes can be observed. The peak intensities of the diffraction spots were sufficiently strong to be detected. For example, the (020) spot intensity was about 50 times higher than the rms background noise of the image. In multiple-shot measurement, the clarity of the image was improved. As shown in Fig. 3(b), second-order diffraction spots of (040) became clearly visible with ten-shot accumulation. From the distance of the diffraction spots and the known lattice parameters of $\mathrm{Au}$, the electron momentum was estimated to be $680 \mathrm{keV} / \mathrm{c}$ (340 keV in energy), which is in fairly good agreement with the present simulation result.

In summary, we have demonstrated the imaging of single-shot picosecond electron diffraction with sub-MeV electron pulses generated by acceleration in plasma produced by intense femtosecond laser pulses. Hence, the laseraccelerated electron pulse beam is a very promising candidate for an intense electron source for UED. The generated electron beam is highly reversible in both the transverse and longitudinal directions since the fairly good agreement between the numerical simulations and the measurement results of the transverse beam profile supports the assumptions that there is no space-charge effect around the electron emission source and that the emission area is comparable in size to the laser-irradiated area. The brightness of the electron source needs to be increased by one order of magnitude to accommodate various applications. However, taking into account that the conditions of laser irradiation and target have not been optimized in the present experiment and that the brightness will be improved through various approaches such as increasing the laser pulse energy, laser-accelerated electron sources have a high potential for use in single shot UED.

We thank Professor Seiji Isoda, Professor Hiroki Kurata, and Professor Takashi Nemoto of Kyoto University for their valuable suggestions. This work was supported by a Grantin-Aid for Scientific Research (A) (Grant No. 18206006) and a Grant-in-Aid for the Global COE Program "The Next Generation of Physics, Spun from Universality and Emergence" from the Ministry of Education, Culture, Sports, Science and Technology (MEXT), Japan.

${ }^{1}$ R. Srinivasan, V. A. Lobastov, C.-Y. Ruan, and A. H. Zewail, Helv. Chim. Acta 86, 1761 (2003).

${ }^{2}$ W. E. King, G. H. Campbell, A. Frank, B. Reed, J. F. Schmerge, B. J. Siwick, B. C. Stuart, and P. M. Weber, J. Appl. Phys. 97, 111101 (2005). ${ }^{3}$ J. R. Dwyer, C. T. Hebeisen, R. Ernstorfer, M. Harb, V. B. Deyirmenjian, R. E. Jordan, and R. J. D. Miller, Philos. Trans. R. Soc. London, Ser. A 364, 741 (2006).

${ }^{4}$ A. H. Zewail, Annu. Rev. Phys. Chem. 57, 65 (2006).

${ }^{5}$ M. Dantus, S. B. Kim, J. C. Williamson, and A. H. Zewail, J. Phys. Chem. 98, 2782 (1994).

${ }^{6}$ J. Cao, Z. Hao, H. Park, C. Tao, D. Kau, and L. Blaszczyk, Appl. Phys. Lett. 83, 1044 (2003).

${ }^{7}$ B. J. Siwick, J. R. Dwyer, R. E. Jordan, and R. J. D. Miller, Science 302, 1382 (2003).

${ }^{8}$ M. Harb, R. Ernstorfer, C. T. Hebeisen, G. Sciaini, W. Peng, T. Dartigalongue, M. A. Eriksson, M. G. Lagally, S. G. Kruglik, and R. J. D. Miller, Phys. Rev. Lett. 100, 155504 (2008).

${ }^{9}$ G. Sciaini, M. Harb, S. G. Kruglik, T. Payer, C. T. Hebeisen, F.-J. M. zu Heringdorf, M. Yamaguchi, M. H. Hoegen, R. Ernstorfer, and R. J. D. Miller, Nature (London) 458, 56 (2009).

${ }^{10}$ P. Baum and A. H. Zewail, Proc. Natl. Acad. Sci. U.S.A. 103, 16105 (2006).

${ }^{11}$ T. van Oudheusden, E. F. de Jong, S. B. van der Geer, W. P. E. M. Op 't Root, O. J. Luiten, and B. J. Siwick, J. Appl. Phys. 102, 093501 (2007).

${ }^{12}$ P. Kung, H. Lihn, H. Wiedemann, and D. Bocek, Phys. Rev. Lett. 73, 967 (1994).

${ }^{13}$ S. G. Anderson, P. Musumeci, J. B. Rosenzweig, W. J. Brown, R. J. England, M. Ferrario, J. S. Jacob, M. C. Thompson, G. Travish, A. M. Tremaine, and R. Yoder, Phys. Rev. ST Accel. Beams 8, 014401 (2005).

${ }^{14}$ J. B. Hastings, F. M. Rudakov, D. H. Dowell, J. F. Schmerge, J. Cardoza, J. M. Castro, S. M. Gierman, H. Loos, and P. M. Weber, Appl. Phys. Lett. 89, 184109 (2006).

${ }^{15}$ D. W. Forslund, J. M. Kindel, and K. Lee, Phys. Rev. Lett. 39, 284 (1977)

${ }^{16}$ G. Pretzler, Th. Schlegel, E. Fill, and D. Eder, Phys. Rev. E 62, 5618 (2000).

${ }^{17}$ E. E. Fill, S. Trushin, R. Bruch, and R. Tommasini, Appl. Phys. B: Lasers Opt. 81, 155 (2005).

${ }^{18}$ F. Brunel, Phys. Rev. Lett. 59, 52 (1987).

${ }^{19}$ W. L. Kruer and K. Estabrook, Phys. Fluids 28, 430 (1985).

${ }^{20}$ S. C. Wilks, W. L. Kruer, M. Tabak, and A. B. Langdon, Phys. Rev. Lett. 69, 1383 (1992).

${ }^{21}$ J. van Tilborg, C. B. Schroeder, C. V. Filip, Cs. Tóth, C. G. R. Geddes, G. Fubiani, R. Huber, R. A. Kaindl, E. Esarey, and W. P. Leemans, Phys. Rev. Lett. 96, 014801 (2006).

${ }^{22}$ R. Tommasini, E. E. Fill, R. Bruch, and G. Pretzler, Appl. Phys. B: Lasers Opt. 79, 923 (2004).

${ }^{23}$ X. Wang, K. Nishikawa, and K. Nemoto, Phys. Plasmas 13, 080702 (2006).

${ }^{24}$ Y. T. Li, M. H. Xu, X. H. Yuan, W. M. Wang, M. Chen, Z. Y. Zheng, Z. M. Sheng, Q. Z. Yu, Y. Zhang, F. Liu, Z. Jin, Z. H. Wang, Z. Y. Wei, W. Zhao, and J. Zhang, Phys. Rev. E 77, 016406 (2008).

${ }^{25}$ S. Tokita, M. Hashida, S. Masuno, S. Namba, and S. Sakabe, Opt. Express 16, 14875 (2008)

${ }^{26}$ General Particle Tracer, http://www.pulsar.nl/gpt/. 